\title{
Properties of Rod-Based Artificial Pinning Center Nb-Ti Superconductors
}

\author{
Robert W. Heussner, Paul D. Jablonski, Peter J. Lee and David C. Larbalestier \\ Applied Superconductivity Center, University Of Wisconsin-Madison, USA
}

\begin{abstract}
We have fabricated rod based artificial pinning center (APC) composites from rods of $\mathrm{Nb}-\mathrm{Ti}$ and $\mathrm{Nb}$ as matrix and pinning center respectively. Critical current densities of $19000 \mathrm{~A} / \mathrm{mm}^{2}$ at $1 \mathrm{~T}, 12500 \mathrm{~A} / \mathrm{mm}^{2}$ at $2 \mathrm{~T}$ and $3000 \mathrm{~A} / \mathrm{mm}^{2}$ at 5 $\mathrm{T}$ were achieved in a conductor containing $\mathrm{Nb} 47 \mathrm{wt} . \% \mathrm{Ti}$ with 24 vol\% Nb pins. Microscopy revealed a progressive degradation of the original hexagonal pinning centers from an ordered array towards a more random ribbon-type microstructure as the composites were drawn towards their peak $\mathbf{J}_{c^{*}}$ We review the impact of this deformation on the physical properties.
\end{abstract}

\section{INTRODUCTION}

In recent years, the maximum critical current density $\left(\mathrm{J}_{\mathrm{c}}\right)$ of conventionally processed $\mathrm{Nb}-\mathrm{Ti}$ superconducting wires has not increased significantly. High $\mathrm{J}_{\mathrm{c}}$ values are attained through a thermo-mechanical treatment of the alloy which serves to develop a fine dispersion of $\alpha$-Ti precipitates [1]. The precipitates are then refined into ribbons during subsequent wire drawing. Optimum flux pinning occurs with ribbons 1-2 $\mathrm{nm}$ thick separated by 3-6 $\mathrm{nm}$ [1], [2]. The highest $\mathbf{J}_{\mathrm{c}}(5 \mathrm{~T}, 4.2 \mathrm{~K})$ obtained using the conventional process is $\sim 3700 \mathrm{~A} / \mathrm{mm}^{2}$ for $\mathrm{Nb} 46.5 \mathrm{wt} . \% \mathrm{Ti}$ [3]; further increases are limited by the amount of $\alpha-\mathrm{Ti}$ which can be precipitated from the alloy. $J_{c}$ increases linearly with volume fraction of $\alpha$-Ti up to at least 25\% [4]; larger amounts cannot be attained in $\mathrm{Nb} 47 \mathrm{wt} . \% \mathrm{Ti}$. This ceiling on the amount of pinning center is unfortunate, for Stejic et al. [5] predicted further increases in $\mathrm{J}_{\mathrm{c}}$ for a microstructure containing $40 \mathrm{vol} \% \alpha-\mathrm{Ti}$. Clearly, there is an incentive to develop new nanostructures having a higher amount of pinning center.

Several methods for creating artificial dispersions of pinning centers in the $\mathrm{Nb}-\mathrm{Ti}$ microstructure have been used. However, the majority of these methods require fabrication strains of order 30 and multiple multifilament stacks. In order for APC conductors to be cost effective for use in high field magnets, they must demonstrate substantially increased critical current densities over the conventional process at mid to high fields (5-8 $\mathrm{T})$ and favorable fabrication properties.

Several groups [6]-[11] have fabricated APC conductors using different fabrication techniques which achieve high critical current densities at low fields (1-5 T); most methods

Manuscript received October 16, 1994

This work was supported in part by the Department of Energy-Division of High Energy Physics under grant \#DF-FGO2-91 ER40643. use $\mathrm{Nb}$ as the pinning material. Recently, a record 4250 $\mathrm{A} / \mathrm{mm}^{2}(5 \mathrm{~T}, 4.2 \mathrm{~K})$ without orientational anisotropy [9] was attained by Matsumoto et al. for a $\mathrm{Nb} 50 \mathrm{wt} . \% \mathrm{Ti}$ with a 27 vol\% $\mathrm{Nb}$ multilayered APC structure. However, the $\mathrm{J}_{\mathrm{c}}(7 \mathrm{~T})$ for this composite reached only $60 \%$ of that obtained in conventionally processed material. The fall off in $\mathrm{J}_{\mathrm{c}}$ at high fields was attributed to a suppressed $B_{c 2}$ due to proximity coupling of the matrix with the $\mathrm{Nb}$ layers. Although critical current densities in APC composites are increasing, even exceeding those for the conventional process, there currently exists little hard information on their microstructuresuperconducting properties relationships. Most $J_{c}$ data are correlated to a calculated pin diameter extrapolated from sizes much larger than that at which peak $J_{c}$ is found. Both the size and shape for the pins may deviate substantially from the true microstructure.

Many factors can influence flux pinning in APC composite superconductors including the pinning center composition, shape and fraction, as well as the optimum matrix composition for a given pin arrangement. Since making one APC composite can be very cost and labor intensive, it is advantageous to use a fabrication scheme which minimizes the overall process complexity and expense. An APC fabrication technique which uses rods of both the superconductor and the pinning center to create artificial pinning structures has been investigated by two groups [12], [13]. Using this method the pin and matrix compositions, as well as pin geometry, can be varied easily. Once the initial APC geometry is formed, the ratio of pin diameter to filament diameter and the number of filaments and their spacing can be controlled by the subsequent stacking methods. In addition, rod material is widely available and assembly is relatively simple, making it practical to fabricate many different pinning structures. The present paper describes the superconducting properties of such a rod-based APC conductor containing Nb $47 \mathrm{wt} . \% \mathrm{Ti}$ with $24 \mathrm{vol} \% \mathrm{Nb}$ pins.

\section{EXPERIMENTAL PROCEDURE}

The rod-based APC conductor was fabricated from 96 rods of $\mathrm{Nb} 47 \mathrm{wt} . \% \mathrm{Ti}$ and 31 rods of $\mathrm{Nb}$ assembled in a hexagonal stack, each rod measuring $1 \mathrm{~mm}$ in diameter and $\sim 8 \mathrm{~cm}$ long. The $\mathrm{Nb}$ pins were arranged in a hexagonal pattern within the stack with the pin diameter $d_{r}$ approximately equal to the pin spacing $d_{s}$. The stack was encased in a $1.6 \mathrm{~cm}$ OD copper extrusion can and a nose cone, equipped with an evacuation tube, was arc-welded in 

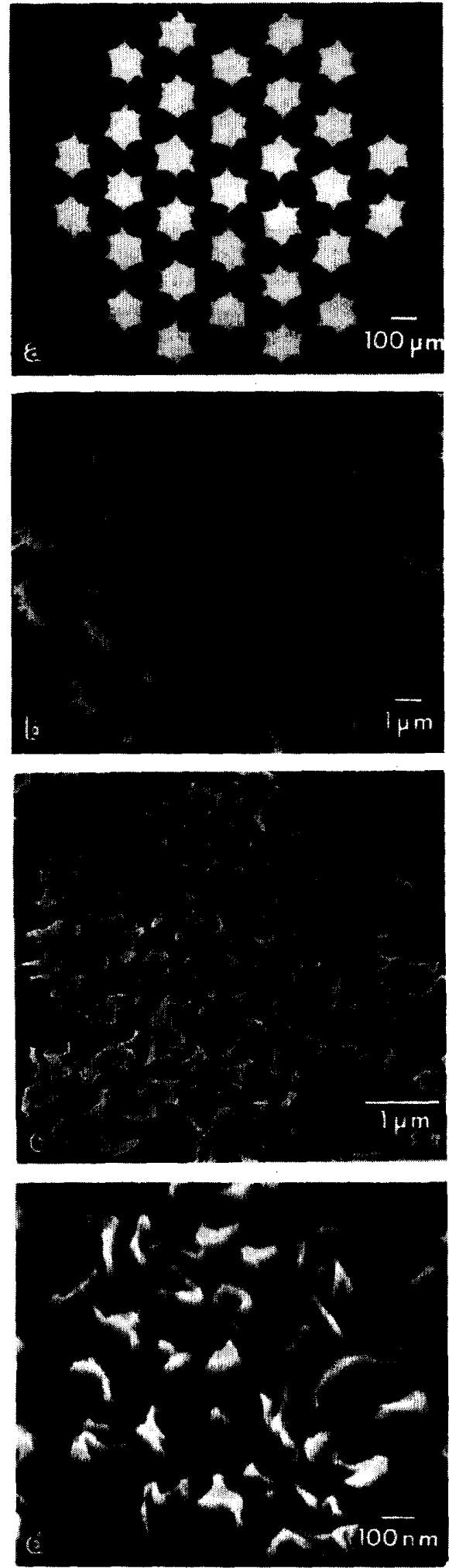

Fig. 1. (a) SEM-backscattered electron micrograph of the first extrusion composite showing a pinning center array at $d_{p} \sim 100 \mu \mathrm{m}$ (b) SEM-secondary electron micrograph of the third extrusion composite at $d_{p} \sim 3 \mu \mathrm{m}$. (c) FESEM-secondary electron micrograph of the fourth extrusion composite at $d_{p} \sim 600 \mathrm{~nm}$ and (d) $d_{p} \sim 100 \mathrm{~nm}$. Note that the pinning center shape changes from nominally hexagonal to ribbons with an aspect ratio of $~ 3-10: 1$.

an enclosed argon atmosphere. Following evacuation to $<5 \times 10^{-3} \mathrm{~Pa}$, the billet was sealed, preheated to $650^{\circ} \mathrm{C}$, and hydrostatically extruded using an area reduction ratio of 15 . The extruded wire was drawn to $1.6 \mathrm{~mm}$ diameter, cut into 127 pieces and the copper was chemically removed. The $\mathrm{Nb}-\mathrm{Ti} / \mathrm{Nb}$ filaments were stacked in a hexagonal array, recanned in $\mathrm{Cu}$ and extruded using identical processing conditions. The restacking and extrusion sequence was repeated a third time, the product being a monofilament containing 499999 pinning centers. Following the third extrusion, part of the wire was drawn to $0.08 \mathrm{~mm}$ diameter and the rest was cut into seven pieces, the $\mathrm{Cu}$ removed and another monofilament stack was assembled and extruded. This fourth extrusion composite was also drawn to $0.08 \mathrm{~mm}$ diameter.

Critical current measurements were made on wire diameters ranging from $0.3 \mathrm{~mm}$ to $0.08 \mathrm{~mm}$, using a $10^{-14}$ $\Omega \mathrm{m}$ criterion with voltage taps spaced $22-33 \mathrm{~cm}$ apart. This wire diameter range corresponds to a $d_{p}$ range of 165 $\mathrm{nm}$ to $40 \mathrm{~nm}$ for the three extrusion composite and $60 \mathrm{~nm}$ to $15 \mathrm{~nm}$ for the fourth extrusion composite. Here, the pinning center diameter $d_{p}$ refers to the effective pin diameter, as calculated using the relationship:

$$
d_{p}=\frac{d_{w}}{\sqrt{N(1+R)}}
$$

where $d_{w}$ is the wire diameter, $N$ is the number of rods in the composite (i.e. $127,16129,2048383$, and 14338681 for the first through fourth extrusions respectively), and $R$ is the volume ratio of copper to superconductor. Copper to superconductor ratios were determined using the weigh and etch technique and the calculated density of the $\mathrm{Nb}-\mathrm{Ti} / \mathrm{Nb}$ composite. The composite wires were analyzed using Scanning Electron Microscopy (SEM) and Field Emission SEM (FESEM).

\section{RESULTS}

The microstructural development of the $\mathrm{Nb}$ pinning centers as a function of drawing reduction is shown in Fig. 1. Fig. 1a shows a Nb pinning center array in the first extrusion composite where $d_{p} \sim 100 \mu \mathrm{m}$. The pinning center shape is nominally hexagonal with an irregular surface. At $d_{p} \sim 3 \mu \mathrm{m}$ (Fig. 1b) the degree of surface irregularity has increased. At $\mathrm{d}_{\mathrm{p}} \sim 600 \mathrm{~nm}$ (Fig. 1c) the $\mathrm{Nb}$ pins are becoming shape unstable. By $d_{p} \sim 100 \mathrm{~nm}$ (Fig. 1d) the pins 


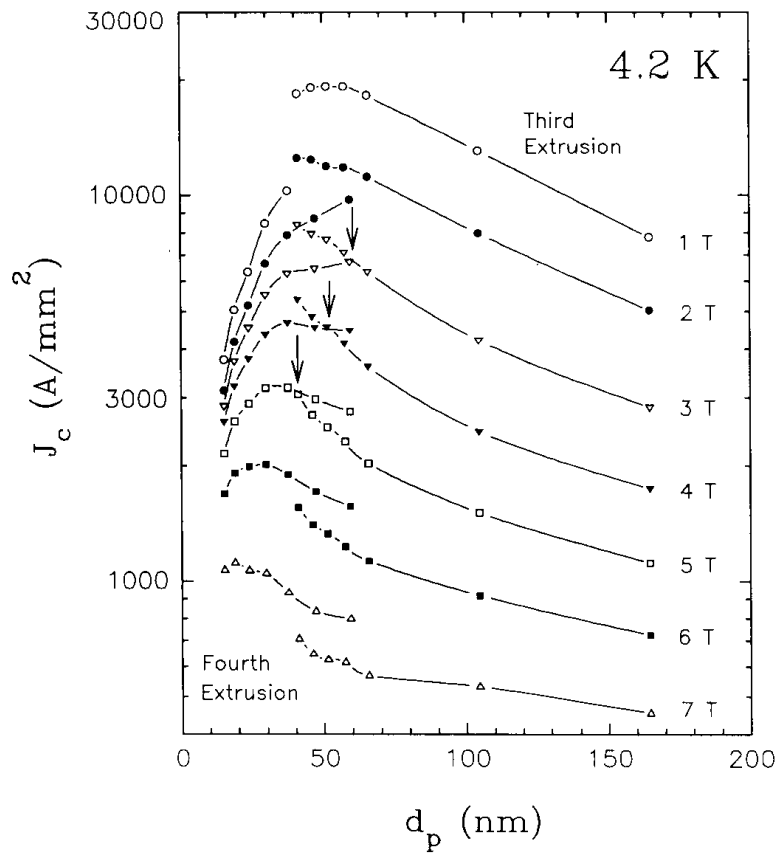

Fig 2. $J_{c}$ versus $d_{f}$ curves as a function of magnetic field at $4.2 \mathrm{~K}$ for the third and fourth extrusion composites where $d_{p}$ varies from $\sim 165 \mathrm{~nm}$ to $15 \mathrm{~nm}$. The three arrows indicate the cross over size, $d_{p}$, between the two composites decreases with increasing applied field. Note that the real pin thickness is smaller than $d_{p}$.

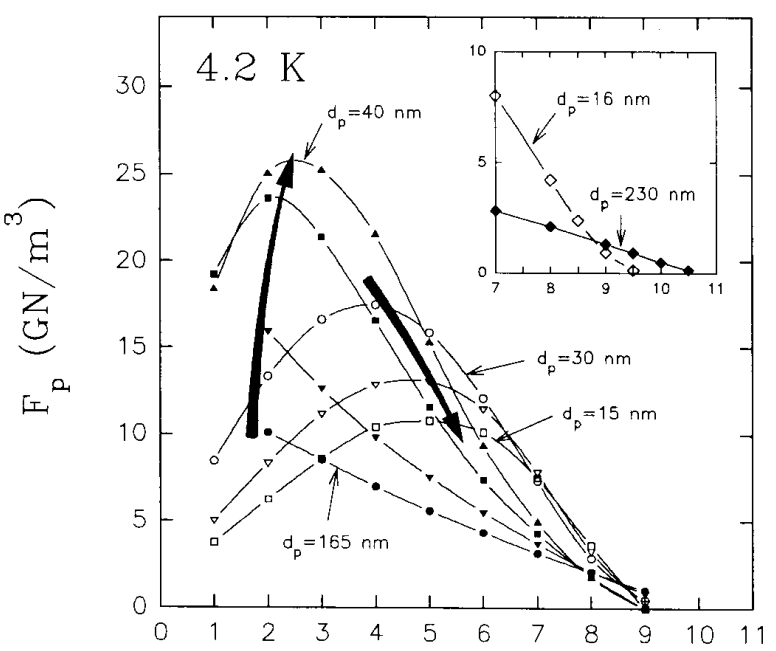

$\mathrm{B}(\mathrm{T})$

Fig 3. $F_{p}$ versus $B$ curves as a function of the effective pinning center diameter, $d_{p}$. The insert shows more detail of the high field $F_{p}$ curves for a similar composite ( $\mathrm{Nb} 47 \mathrm{wt} \% \mathrm{Ti}$ with 21 vol\% $\mathrm{Nb}$ ). Filled and open symbols correspond to third and fourth extrusion composites respectively. Note the apparent depression in $\mathrm{B}_{\mathrm{c} 2}$ as the pinning center size decreases from $230 \mathrm{~nm}$ to $16 \mathrm{~nm}$. have developed into ribbons, with aspect ratios ranging from -3-10:1.

$J_{c}$ versus $d_{p}$ is shown for applied fields from 1-7 T in Fig. 2. The two sets of curves represent the third and fourth extrusion composites. The set from $d_{n} \sim 165-40 \mathrm{~nm}$ is for the third extrusion composite, another set from $d_{p} \sim 60-15 \mathrm{~nm}$ is for the fourth extrusion composite. The peak $\mathbf{J}_{\mathrm{c}}$ at $1 \mathrm{~T}$ of $19000 \mathrm{~A} / \mathrm{mm}^{2}$ occurs at $d_{n} \sim 50 \mathrm{~nm}$; at $7 \mathrm{~T}$ the peak value of $1100 \mathrm{~A} / \mathrm{mm}^{2}$ occurs at $d_{n} \sim 19 \mathrm{~nm}$. Between $d_{p} \sim 60-40 \mathrm{~nm}$, the region where the two data sets overlap, no curve alignment is seen. There is not a proportional difference between the two sets of curves, but rather, the difference changes systematically with applied magnetic field. At 1-3 $\mathrm{T}$ the third extrusion composite has significantly higher $\mathrm{J}_{\mathrm{c}}$ values than the fourth extrusion composite in the range of $\mathrm{d}_{\mathrm{p}} \sim 60-40 \mathrm{~nm}$. However, between 3 and $5 \mathrm{~T}$, a crossover occurs, as shown by the three vertical arrows in Fig. 2, and at higher fields (5-7 T) the fourth extrusion composite $\mathrm{J}_{\mathrm{c}}$ values exceed those for the third extrusion composite.

Fig. 3 shows bulk flux pinning force $\left(F_{p}\right)$ versus applied magnetic field curves as a function of the effective pin diameter. The arrows indicate the directions of decreasing pin diameter and they intersect the peak value for each curve. At larger pin sizes $\left(d_{p}=165 n m\right)$ the $F_{p}$ curve has a shallow slope, decreasing with applied field. As the pin size decreases the curve increases in magnitude at lower fields, developing a $2 \mathrm{~T}$ peak at $d_{p}=60 \mathrm{~nm}$. A further decrease in pin size to $40 \mathrm{~nm}$ shifts the peak to $2-3 \mathrm{~T}$ and the maximum $F_{p}$ to $25 \mathrm{GN} / \mathrm{m}^{3}$. Further decreases in $d_{p}$ shift the peak in $F_{p}$ towards higher fields while reducing the peak magnitude. The inset graph in Fig. 3 shows a depression in $\mathrm{B}_{\mathrm{c} 2}$ from $\sim 10.5$ to $9.5 \mathrm{~T}$ as $\mathrm{d}_{\mathrm{r}}$ changes from 230 to $16 \mathrm{~nm}$ for a similar design of APC composite ( $\mathrm{Nb} 47$ wt.\% Ti with 21 vol\% $\mathrm{Nb})$.

\section{DISCUSSION}

Changes in the shape of the pin with amount of reduction can be explained by the bcc metal $\langle 110\rangle$ drawing texture which develops in $\mathrm{Nb}$ and $\mathrm{Nb}$-Ti. [14], [15]. During drawing, bcc grains deform so that a $<110\rangle$ direction is aligned parallel to the drawing axis. This allows for slip in only two of four $<111>$ directions. Plane strain conditions then prevail, deforming the grains into elongated ribbons which intercurl to maintain grain boundary continuity. It is this grain intercurling which leads to the degradation of the round pinning center shape. This shape change mechanism is also responsible for $\alpha-\mathrm{Ti}$ ribbon development in the conventionally processed microstructure. In the conventional process the $\beta-\mathrm{Nb}-\mathrm{Ti}$ grains control the ribbon formation, whereas here, it is reasonable to suppose that both the $\mathrm{Nb}$ and $\mathrm{Nb}-\mathrm{Ti}$ grain structures determine the $\mathrm{Nb}$ ribbon shape. This parallels the non uniform deformation of $\mathrm{Nb}$ diffusion barriers in the conventional process [16]. 
The fact that the pins are not round at small pin sizes suggests that using the calculated effective pin diameter, $d_{p}$, to correlate the pinning structure to the superconducting properties will be misleading. We are currently investigating the quantification of the microstructure in more detail [17], [18]. In any case it appears that the effective pin thickness is always several times less than $d_{p}$. Thus, care must be taken in making microstructure/ properties relationships based on extrapolated microstructural dimensions.

The critical current densities of samples from the third and fourth extrusion composite are not well behaved in the overlap region. It is curious that the high field $\mathbf{J}_{c}$ was enhanced at the fourth extrusion but that the low field $\mathrm{J}_{\mathrm{c}}$ was distinctly lower than the third. An important clue to this behavior must lie in the microstructure which requires analysis by transmission electron microscopy which has not yet been performed. At present we suppose that nonnegligible interdiffusion occurs at this time since $d_{p}$ at this stage is only $2 \mu \mathrm{m}$. Such interdiffusion should diminish the depth of the strongest pinning wells as seen in the low field data. This explanation evidently does not hold at higher fields, although the changes are not so large.

The depression of $B_{c 2}$ seen in Fig. 3 is a consequence of proximity induced coupling of the $\mathrm{Nb}$ pins with the $\mathrm{Nb}-\mathrm{Ti}$ matrix. At large pin sizes $\left(d_{p}>230 \mathrm{~nm}\right)$ little proximity coupling occurs and the composite $\mathrm{B}_{c 2}$ is governed by the $\mathbf{B}_{\mathrm{c} 2}$ of the matrix ( $10.7-11 \mathrm{~T}$ for $\mathrm{Nb} 47 \mathrm{wt} . \% \mathrm{Ti})$. At small pin sizes $\left(d_{0}<16 \mathrm{~nm}\right)$ the pins are significantly coupled and the $\mathrm{B}_{\mathrm{c} 2}$ tends toward the $\mathrm{B}_{\mathrm{c} 2}$ of the matrix and pin mixture $(\mathrm{Nb}$ 34 wt.\% Ti). This result is consistent with the results of others [7]-[11].

\section{CONCLUSIONS}

A process for assembling rods of both $\mathrm{Nb}-\mathrm{Ti}$ superconductor and pinning center to create uniform pinning microstructures has been examined. This flexible technique has shown to be effective in producing artificial pinning center microstructures. A conductor containing Nb $47 \mathrm{wt} . \%$ Ti with $24 \mathrm{vol} \% \mathrm{Nb}$ pins has achieved $19000 \mathrm{~A} / \mathrm{mm}^{2}$ at $1 \mathrm{~T}$, $8500 \mathrm{~A} / \mathrm{mm}^{2}$ at $3 \mathrm{~T}$, and $3000 \mathrm{~A} / \mathrm{mm}^{2}$ at $5 \mathrm{~T}$. Lower high field $\mathbf{J}_{c}$ values than conventional $\mathrm{Nb}-\mathrm{Ti}$ are attributed to a depression in $\mathrm{B}_{\mathrm{c} 2}$ caused by proximity coupling of the $\mathrm{Nb}$ pins to the Nb-Ti matrix.

\section{ACKNOWLEDGMENTS}

Support was provided in part by the IMR, Madison, WI, through NIH Biomedical Research Technology Grant RR 00570. We wish to thank W. Starch, L. Lincoln, J. Marquardt, D. Ohmann, and Y. Chen for experimental assistance.

\section{REFERENCES}

[1] P.J. Lee and D.C. Larbalestier, Acta Metall. 35 (1987) 2523-2536.

[2] C. Meingast, P.J. Lee, and D.C. Larbalestier, J. Appl Phys. 66 (1989) 5962-5970.

[3] Li Chengren and D.C. Larbalestier, Cryogenics 27 (1987) 171-177.

[4] P.J. Lee, J.C. McKinnell, and D.C. Larbalestier, Adv. Cryo. Eng. 36 (1989) 287-294.

[5] G. Stejic, L.D. Cooley, R. Joynt, D.C. Larbalestier, and S. Takacs, Supercond. Sci. Technol. 5 (1992) S177-179.

[6] G.L. Dorofejev, E.Y. Klimenko, S.V. Froloy, E.V. Nikulenkov, E.I. Plashkin, N.I. Salunin, and V.Y. Filkin, Proc. of 9th Inter. Conf. on Magnet Technology, C. Marinucci and P. Weymuth, Eds. (1985) 564566.

[7] L.R. Motowidlo, B.A. Zeitlin, M. Walker, and P. Haldar, Appl. Phys Lett. 61 (1994) 99I-993.

[8] J. Wong and M.K. Rudziak, U.S. Patent No. 5,223,348 (July 13, 1994)

[9] K. Matsumoto, H. Takewaki, Y. Tanaka, O. Miura, K. Yamafuji, K. Funaki, M. Iwakuma, and T. Matsushita, App. Phys. Lett. 64 (1994) 115-117.

[10] P.D. Jablonski, P.J. Lee, and D.C. Larbalestier, Appl. Phys. Lett. 65 (1994) 767-769.

[11] L.D. Cooley, P.J. Lee, D.C. Larbalestier, and P.M. O'Larey, Appl. Phys. Lett. 64 (1994) 1298-1300.

[12] N. Rizzo, S. Ling, J. McCambridge, D. Prober, L. Motowidlo, and B. Zeitlin, LTS workshop, Devil's Head, WI (Feb., 1994).

[13] R.W. Heussner, P.D. Jablonski, P.J. Lee, and D.C. Larbalestier, LTS workshop, Devil's Head, W1 (Feb., 1994).

[14] J.F. Peck and D.A. Thomas, Trans. Met. Soc. AIME 221 (1961) 12401247.

[15] W.F. Hosford, Trans. Met. Soc. AIME 230 (1964) 12-15.

[16] R.W. Heussner, P.J. Lee, P.D. Jablonski, and D.C. Larbalestier, Adv. Cryo. Eng. 40 (1994) 755-762.

[17] P.J. Lee, P.D. Jablonski, and D.C. Larbalestier, paper MQA-4, this conference.

[18] P.D. Jablonski, Ph.D. thesis, UW-Madison (1994). 